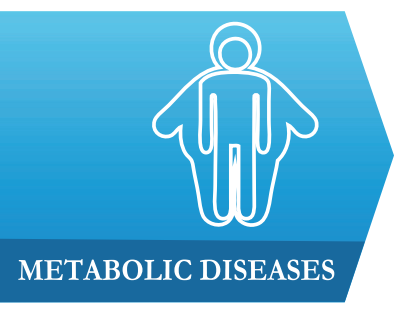

1) Department of Diabetes and Nutrition Diseases, Faculty of Medicine, Iuliu Hatieganu University of Medicine and Pharmacy, ClujNapoca, Romania

2) Emergency Clinical County Hospital, Central Laboratory, ClujNapoca, Romania

3) Emergency Clinical County Hospital, Centre of Diabetes, Nutrition and Metabolic Diseases, Cluj-Napoca, Romania

DOI: $10.15386 / \mathrm{mpr}-1247$

Manuscript received: 18.12 .2018

Received in revised form: 18.02.2019

Accepted: 08.03.2019

Address for correspondence:

dana.ciobanu@umfcluj.ro

\title{
Glycated albumin is correlated with glycated hemoglobin in type 2 diabetes
}

Dana Mihaela Ciobanu ${ }^{1}$, Florina Bogdan², Cristina-Ioana Pătruț ${ }^{3}$, Gabriela Roman ${ }^{1}$

\begin{abstract}
Background and aims. Glycated hemoglobin $(\mathrm{HbA1c})$ retrospectively evaluates mean glycemia in the preceding 2-3 months and is the gold standard for assessing glycemic control, while glycated albumin (GA) is currently considered a short to intermediate term integrated glycemic control marker, since it reflects glycemic status over the last 3 weeks. We aimed to investigate the levels of GA, HbAlc and fasting glycemia in a group of patients with type 2 diabetes.
\end{abstract}

Methods. The observational study included adult type 2 diabetes patients $(n=135)$ according to inclusion and exclusion criteria, randomly selected from Clinical Centre of Diabetes, Cluj-Napoca, Romania. Fasting glycemia, GA, HbA1c and creatinine were measured using commercially available methods.

Results. Of the whole group, 62 (45.9\%) were men. Mean age was $62.1 \pm 8.6$ years old, body mass index was $31.8 \pm 6.1 \mathrm{~kg} / \mathrm{m} 2$ and diabetes duration was $10.0(4.0 ; 15.0)$ years. Fasting glycemia was $162 \pm 13.7 \mathrm{mg} / \mathrm{dl}$, GA was $28.0(21.0 ; 40.0) \%$ and $\mathrm{HbAlc}$ $8.9 \pm 2.3 \%$. We found GA was significantly correlated with $\mathrm{HbAlc}(\mathrm{r}=0.19 ; \mathrm{p}=0.029)$ and fasting glycemia $(\mathrm{r}=0.32 ; \mathrm{p}<0.001)$, while $\mathrm{HbAlc}$ was significantly correlated with fasting glycemia $(\mathrm{r}=0.40 ; \mathrm{p}<0.001)$.

Conclusions. GA was significantly correlated with both $\mathrm{HbA} 1 \mathrm{c}$ and fasting glycemia in our patients with type 2 diabetes. While $\mathrm{HbA1c}$ is recognized as being the reference test for diabetes control monitoring, GA might a useful biomarker for assessing short to intermediate term glycemic control, particularly important in situations when $\mathrm{HbA} 1 \mathrm{c}$ test cannot be reliable or earlier clinical decision making is mandatory.

Keywords: glycated serum albumin, glycated hemoglobin, type 2 diabetes mellitus

\section{Background and aims}

In patients with diabetes mellitus, diagnosis and follow-up are managed using fasting and two-hour postprandial glycemia and glycated hemoglobin (HbA1c) evaluation [1]. Although HbA1c is the reference test used for long term blood glucose monitoring over the last 2-3 months, its utility has its limits related to certain clinical situations or to analytical methods employed, which may cause false results for $\mathrm{HbAlc}$ that are not truly correlated with the mean glycemia $[2,3]$.

In the latest decades, glycated albumin (GA) gained more attention as being a new parameter for glycaemic control monitoring. GA is a fructosamine formed by non-enzymatic glycation process of serum albumin and it reflects short to intermediate term mean glycaemic levels due to the half life time of the albumin, which is approximately 3 weeks [4-6].
GA levels are not affected by changes in erythrocyte lifespan, and measurement of GA is not influenced by conditions such as hemolytic anaemias or bleeding episodes that might falsely reduce $\mathrm{HbAlc}$ levels, or iron deficiency anemias, thalassemia or other hemoglobinopathies which may falsely elevate $\mathrm{HbA} 1 \mathrm{c}$ levels, invalidating $\mathrm{HbA1c}$ measurements in the management and diagnosis of diabetes. Also, GA can be useful for patients with wide variations in blood glucose, those at higher risk for hypoglycaemia or presenting with advanced chronic kidney disease.

Given the fact that GA represents an intermediate measure between $\mathrm{HbA} 1 \mathrm{c}$ and blood glucose, it might be helpful in clinical decision making, particularly when new antihyperglycemic treatments are initiated. Thus, the use of GA has been proposed as a complementary biomarker for diabetes mellitus monitoring particularly useful 
in short to intermediate term glucose monitoring and in situations when HbA1c test cannot be reliable $[7,8]$. We aimed to investigate the levels of GA, HbAlc and fasting glycemia in a group of patients with type 2 diabetes.

\section{Methods}

\section{Study design and patients}

The observational study included randomly selected adult patients with type 2 diabetes $(n=135)$ hospitalized in Clinical Centre of Diabetes, Nutrition and Metabolic Diseases in Cluj-Napoca, Romania, between years 2013 and 2018. The diagnosis of type 2 diabetes and diabetes chronic complications was established according to the American Diabetes Association criteria [1]. Hypertension was diagnosed in the presence of office BP of $\geq 140 \mathrm{mmHg}$ systolic or $\geq 90 \mathrm{mmHg}$ diastolic or the use of antihypertensive drugs [1]. Patients were not included if they were diagnosed with any unstable clinical conditions, hematological diseases (e.g. anemia, thalassemia), malignancies, nephrotic syndrome, liver cirrhosis, hyperthyroidism, hypothyroidism, had estimated glomerular filtration rate $<30 \mathrm{ml} / \mathrm{min} / 1.73 \mathrm{~m} 2$, had steroid medications, had a blood transfusion within 6 months of study entry, were pregnant or breast feeding. All these conditions might have interfered with study evaluation of HbAlc and GA levels $[3,4]$.

In accordance with the World Medical Association Declaration of Helsinki revised in 2000, Edinburgh, and institutional guidelines, the protocol was approved by the local Ethics Committee of the Iuliu Haţieganu University of Medicine and Pharmacy Cluj-Napoca, Romania. All patients were aware of the investigational nature of the study and provided written informed consent before any study procedure.

\section{Study protocol and assessments}

Age, sex, smoking status, duration of diabetes and hypertension, presence of chronic diabetes complications (neuropathy, retinopathy, nephropathy and atherosclerotic cardiovascular disease), other concomitant diseases and antidiabetic medication were collected from patients' medical files. Height, weight and abdominal circumference were measured in fasting state, light dress and with no shoes and body mass index was calculated. Office systolic and diastolic blood pressure and heart rate were measured in both arms using an automatic device (Colin Press-Mate BP-8800C Sphygmomanometer Monitor, Japan) after 10 minutes of rest in a sitting position.

Fasting venous blood samples were obtained from each patient for the assessment of glycemia, HbAlc, complete blood count and creatinine. Parameters were assessed on the day of blood collection using commercially available methods at Central Laboratory of the Emergency County Hospital Cluj, Romania (Beckman Coulter AU480 Chemistry Analyzer, USA). Glycemia was determined using the hexokinase method and $\mathrm{HbAlc}$ was measured in ethylenediamine tetra-acetic acid (EDTA) anticoagulated blood samples using turbidimetric immunoinhibition method. Serum samples were stored frozen at $-80^{\circ} \mathrm{C}$ for subsequent GA analysis in the year 2018. GA was measured using a standardized enzymatic quantitative method (QuantILab ${ }$, Instrumentation Laboratory SpA - A Werfen Company, Milan, Italy) and was reported as a percentage of total albumin measured in the same serum sample, minimizing the effects of variations among patients and in albumin concentrations. The percentage of GA was obtained by a math calculation: glycated albumin/total albumin $x 100$ [9]. Creatinine was measured by a colorimetric method and glomerular filtration rate was estimated using CKD-EPI equation by accessing www.mdrd.com. Calibrators and controls used during laboratory assessments were utilized according to manufacturer's instructions. The coefficients of variation of the used reagents and instruments were less than $2 \%$.

\section{Statistical analysis}

Data analysis was performed using the IBM SPSS Statistics for Windows, Version 22.0 (Armonk, NY: IBM Corp). Kolmogorov-Smirnov test was used to test the normal distribution of all continuous variables. Continuous variables were tested for outlier using the outlier labelling rule based on multiplying Interquartile Range by a factor of 2.2. Data were expressed as mean \pm standard deviation or median and 25th and 75th percentiles, or numbers and percentages. Correlations between parameters were evaluated using Spearman or Pearson coefficients. A value of $\mathrm{p}<0.05$ was considered statistically significant.

\section{Results}

\section{Characteristics of the study participants}

The characteristics of the study participants are presented in Table I.

Table I. Characteristics of the study participants.

\begin{tabular}{lll}
\hline Variables & Study group (n=135) \\
Age (years) & $62.1 \pm 8.6$ \\
Male gender n, (\%) & $62(45.9)$ \\
Currently smoking n, (\%) & $16(11.9)$ \\
Diabetes duration (years) & $10.0(4.0 ; 15.0)$ \\
Hypertension duration (years) & $11.0(6.0 ; 17.0)$ \\
Office systolic blood pressure (mmHg) & $138.1 \pm 19.1$ \\
Office diastolic blood pressure (mmHg) & $82.6 \pm 12.6$ \\
Heart rate (beats/minute) & $76.4 \pm 11.7$ \\
Body mass index (kg/m2) & $31.8 \pm 6.1$ \\
Waist circumference in males (cm) & $108.7 \pm 13.2$ \\
Waist circumference in women (cm) & $107.6 \pm 12.2$ \\
Fasting glycemia (mg/d) & $162 \pm 13.7$ \\
Glycated albumin, GA $(\%)$ & $28.0(21.0 ; 40.0)$ \\
Glycated haemoglobin, HbA1c $(\%)$ & $8.9 \pm 2.3$ \\
Estimated glomerular filtration rate (ml/ & $77.1 \pm 22.1$ \\
min/1.73m2) & \\
Values are means $+/-$ standard deviation or median and 25th and 75 th \\
percentiles or numbers and percentages.
\end{tabular}


Hypertension was diagnosed and treated with antihypertensive medication in 118 (87.4\%) patients; dyslipidemia was diagnosed in $126(93.3 \%)$ patients, while overweight or obesity was present in $121(89.6 \%)$ patients enrolled in the study. Patients were treated with oral antidiabetic drugs $(n=33,24.4 \%)$, insulin $(n=85,63.0 \%)$ or were not receiving any antihyperglycemic treatment when enrolled $(\mathrm{n}=17,12.6 \%)$. There were no significant changes in chronic antidiabetic medication reported in the 3 months prior to hospital admission, except for insulin dose adjustments according to glycemia levels.

The most frequent chronic microvascular

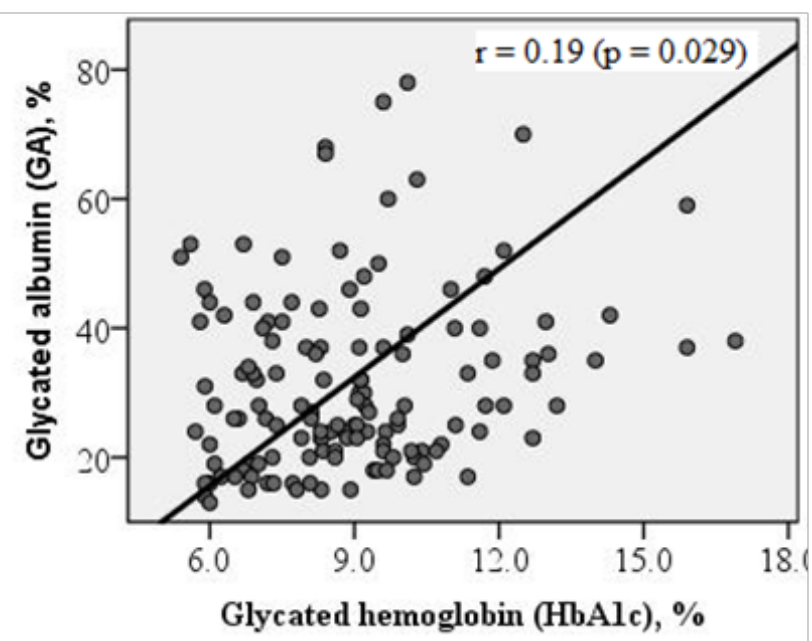

Figure 1. Correlation of glycated albumin (GA) with glycated hemoglobin (HbA1c). complication in the studied population was peripheral neuropathy $(54.3 \%)$, while retinopathy $(28.9 \%)$ and nephropathy (34.8\%) were present in lower percentages. Atherosclerotic cardiovascular disease was present in $46.7 \%$ of the study population.

\section{Correlations}

The correlations between GA, HbAlc and fasting glycemia are presented in Figure 1, Figure 2 and Figure 3. We found that GA significantly correlated with $\mathrm{HbA} 1 \mathrm{c}$ $(r=0.19 ; p=0.029)$ and fasting glycemia $(r=0.32 ; p<0.001)$, while $\mathrm{HbA1c}$ significantly correlated with fasting glycemia $(r=0.40 ; p<0.001)$ and.

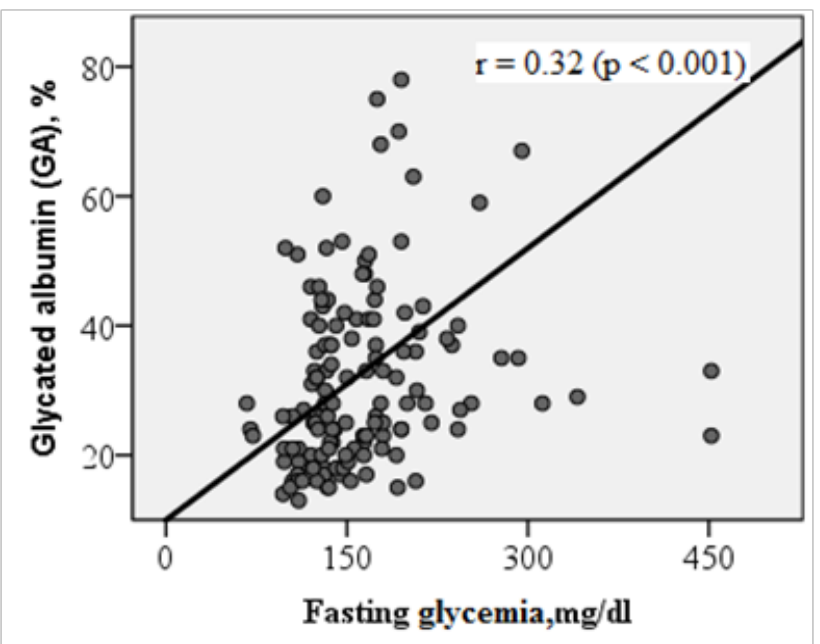

Figure 2. Correlation of glycated albumin (GA) with fasting glycemia.

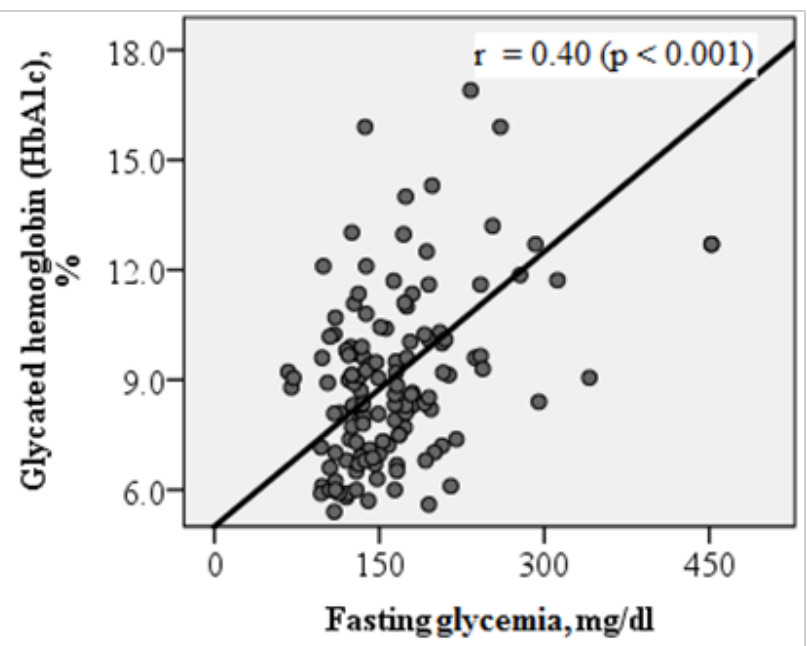

Figure 3. Correlation of glycated hemoglobin (HbA1c) with fasting glycemia. 


\section{Discussion}

We found that GA significantly correlated with both $\mathrm{HbAlc}$ and fasting glycemia in the patients with type 2 diabetes included in our observational study. This significant correlation found between GA and $\mathrm{HbA} 1 \mathrm{c}$ confirms that GA is an indicator of protein glycation as good as $\mathrm{HbA1c}$, and that GA is directly dependent on protein exposure to glucose. GA and $\mathrm{HbA} 1 \mathrm{c}$ are both parameters that reflect blood glucose control but over different time periods. The measurement of $\mathrm{HbAlc}$ is considered the gold standard for monitoring mean glycemia over the last 2-3 months [1], while GA reflects the glycaemic control over the last 3 weeks [4]. Previous studies have shown that GA correlated with both HbA1c and blood glucose values, and that short term assessment of glycemia may be useful as a complement to $\mathrm{HbA1c}$ measurement [10-12].

The low strength of the correlation between GA and $\mathrm{HbA} 1 \mathrm{c}$ we report in our study could be explained by the time period of the glycemic control reflected by each of these parameters. This is thought to be because GA reflects a shorter-term status of glycemic control compared to $\mathrm{HbA1c}$ [10]. In a prospective study, variations in GA appeared to be more marked than those of $\mathrm{HbAlc}$, while the highest GA levels were not consistent with those of HbA1c levels [13]. However, a strong correlation between $\mathrm{HbA1c}$ and GA was reported in cross-sectional studies involving participants without known diabetes [14] and in those with type 2 diabetes [15].

The low strength correlation we found between GA and $\mathrm{HbAlc}$ could have been further investigated if data regarding postprandial blood glucose would have been available for the patients included in our study. It has been suggested that GA may more strongly reflect postprandial glycemia and range of plasma glucose fluctuations than HbAlc [13]. This finding can be explained by the fact that short-term worsening or improvement in glycemic control might be better reflected by GA than by $\mathrm{HbA} 1 \mathrm{c}$ fluctuations. Nathan et al. found that mean pre- and postprandial glycemia means were approximately equally significant associated with both GA and HbA1c. However, fasting glycemia displayed a stronger association with both glycation products than individual pre- or postprandial values [11]. We also found a positive correlation between fasting glycemia, GA and HbA1c. Since mean postprandial glycemia was not uniformly reported for patients included our study, we could not assess the correlation between this parameter, GA and $\mathrm{HbAlc}$.

We should mention that our study population was included based on certain criteria in order to avoid false elevated or false decreased levels of both $\mathrm{HbAlc}$ and GA. It is well known that the presence of certain diseases (e.g. advanced chronic kidney disease, anemia and thalassemia) or physiological conditions (e.g. pregnancy) might influence HbA1c levels, while other diseases might impair GA levels (e.g. nephrotic syndrome, liver cirrhosis, hyperthyroidism and hypothyroidism) $[3,6]$. However, GA could be an additional tool for evaluating blood glucose in pregnant women, new-borns, and patients with advanced chronic kidney disease or on dialysis because it reflects the status of mean glycemia more rapidly than HbA1c [16,17].

Strict glycaemic control in patients with diabetes is known to be associated with lower incidence of chronic microvascular and macrovascular complications. Intensive treatment with insulin or oral antihyperglycemic drugs has been established to delay the onset and to slow the progression of microangiopathy in patients with type 1 and type 2 diabetes included in the Diabetes Control and Complications Trial (DCCT) and United Kingdom Prospective Diabetes Study, respectively $[18,19]$. In these two large prospective studies, lower levels of $\mathrm{HbAlc}$ were significantly associated with lower incidence of chronic complications. Later on, in the DCCT study and its continuation, Epidemiology of Diabetes Interventions and Complications (EDIC) study, HbAlc and GA showed similar associations with microvascular complications [20]. When investigating the interrelationships among different measures of glycemia chronic complications, Nathan et al. reported that $\mathrm{HbA} 1 \mathrm{c}$ and GA had similar associations with retinopathy and nephropathy, and the association was strengthened when both glycation products were considered together [11]. In the Atherosclerosis Risk in Communities (ARIC) study that included patients with type 2 diabetes, GA was strongly associated with diabetes microvascular complications, with prognostic value comparable to $\mathrm{HbAlc}$ [11]. In our observational study, we could only evaluate the presence of chronic diabetes complications as well as the levels of GA and $\mathrm{HbA} 1 \mathrm{c}$, but we could not find any relevant associations between chronic complications and these parameters.

An important aspect related to clinical decision making after glucose-lowering therapy initiation should be mentioned when assessing the differences between $\mathrm{HbAlc}$ and GA. Recent studies have shown that the prediction of therapy success was superior for GA compared to HbA1c when evaluating glycaemic control at an early stage after starting a new glucose-lowering treatment $[21,22]$. Given these findings, it was suggested that GA might be a useful complementary biomarker for assessing glucose control, particularly in situations when earlier clinical decision making is necessary. In our observational study, we evaluated the blood glucose control parameters and antidiabetic medication when patients were included in the study and no previous or follow-up data are reported. Our observational study has certain limitations: the population sample size was restricted to a certain number of available GA measurements and the lack of consistent data regarding postprandial glycemia.

\section{Conclusions}

GA significantly correlated with both $\mathrm{HbA1c}$ and fasting glycemia in patients with type 2 diabetes. While HbAlc is recognized as being the reference test 
for diabetes mellitus control monitoring, GA might be a useful complementary biomarker for short to intermediate term blood glucose fluctuations, particularly important in situations when HbAlc test may be biased or even unreliable, or earlier clinical decision making is mandatory.

\section{Acknowledgements}

The measurement of glycated albumin was funded by Medist S.A, Romania.

\section{References}

1. American Diabetes Association: Standards of Medical Care in Diabetes - 2016. Diabetes Care 2016;39(Supplement1):S1-S112. 2. Sacks DB, Bruns DE, Goldstein DE, Maclaren NK, McDonald JM, Parrott M. Guidelines and recommendations for laboratory analysis in the diagnosis and management of diabetes mellitus. Clin Chem. 2011;57:e1-e47.

3. Cavagnolli G, Pimentel AL, Freitas PA, Gross JL, Camargo JL. Factors affecting A1C in non-diabetic individuals: Review and meta-analysis. Clin Chim Acta. 2015;445:107-114.

4. Koga M. Glycated albumin; clinical usefulness. Clin Chim Acta. 2014;433:96-104.

5. Furusyo N, Hayashi J. Glycated albumin and diabetes mellitus. Biochim Biophys Acta. 2013;1830:5509-5514.

6. Koga M, Kasayama S. Clinical impact of glycated albumin as another glycemic control marker. Endocr J. 2010;57:751-762.

7. Kosecki SM, Rodgers PT, Adams MB. Glycemic monitoring in diabetics with sickle cell plus beta-thalassemia hemoglobinopathy. Ann Pharmacother. 2005;39:1557-1560.

8. Freitas PAC, Ehlert LR, Camargo JL. Glycated albumin: a potential biomarker in diabetes. Arch Endocrinol Metab. 2017;61:296-304.

9. Paleari R, Bonetti G, Callà C, Carta M, Ceriotti F, Di Gaetano $\mathrm{N}$, et al. Multicenter evaluation of an enzymatic method for glycated albumin. Clin Chim Acta. 2017;469:81-86.

10. Koga M, Suzuki S, Matsuo K, Tanahashi Y, Azuma H, Kasayama S. Calculation of HbAlc and glycated albumin from serially measured self-monitored blood glucose in patients with type 1 diabetes mellitus. Clin Chim Acta. 2013;425:188-191.

11. Nathan DM, McGee P, Steffes MW, Lachin JM; DCCT/ EDIC Research Group. Relationship of glycated albumin to blood glucose and HbA1c values and to retinopathy, nephropathy, and cardiovascular outcomes in the DCCT/EDIC study. Diabetes. 2014;63:282-290.
12. Testa R, Ceriotti F, Guerra E, Bonfigli AR, Boemi M, Cucchi M, et al. Glycated albumin: correlation to HbA1c and preliminary reference interval evaluation. Clin Chem Lab Med. 2017;55:e31-e33.

13. Koga M, Murai J, Morita S, Saito H, Kasayama S. Comparison of annual variability in $\mathrm{HbA} 1 \mathrm{c}$ and glycated albumin in patients with type 1 vs. type 2 diabetes mellitus. J Diabetes Complications. 2013;27:211-213.

14. Mo Y, Ma X, Li H, Ran X, Yang W, Li Q, et al. Relationship between glycated albumin and glycated hemoglobin according to glucose tolerance status: A multicenter study. Diabetes Res Clin Pract. 2016;115:17-23.

15. Sato Y, Nagao M, Asai A, Nakajima Y, Takaya M, Takeichi N, et al. Association of glycated albumin with the presence of carotid plaque in patients with type 2 diabetes. J Diabetes Investig. 2013;4:634-639.

16. Gan T, Liu X, Xu G. Glycated Albumin Versus HbA1c in the Evaluation of Glycemic Control in Patients With Diabetes and CKD. Kidney Int Rep. 2017;3:542-554.

17. Huang Y, Hu Y, Ma YU, Ye G. Glycated albumin is an optimal biomarker for gestational diabetes mellitus. Exp Ther Med. 2015;10:2145-2149.

18. Diabetes Control and Complications Trial Research Group, Nathan DM, Genuth S, Lachin J, Cleary P, Crofford O, et al. The effect of intensive treatment of diabetes on the development and progression of long-term complications in insulin-dependent diabetes mellitus. N Engl J Med. 1993;329:977-986.

19. Intensive blood-glucose control with sulphonylureas or insulin compared with conventional treatment and risk of complications in patients with type 2 diabetes (UKPDS 33). UK Prospective Diabetes Study (UKPDS) Group. Lancet. 1998;352:837-853.

20. Selvin E, Rawlings AM, Grams M, Klein R, Sharrett AR, Steffes $M$, et al. Fructosamine and glycated albumin for risk stratification and prediction of incident diabetes and microvascular complications: a prospective cohort analysis of the Atherosclerosis Risk in Communities (ARIC) study. Lancet Diabetes Endocrinol. 2014;2:279-288.

21. Lu JM, Ji LN, Li YF, Li QM, Lin SS, Lv XF, et al. Glycated albumin is superior to glycated hemoglobin for glycemic control assessment at an early stage of diabetes treatment: A multicenter, prospective study. J Diabetes Complications. 2016;30:1609-1613. 22. Desouza CV, Rosenstock J, Zhou R, Holcomb RG, Fonseca VA. Glycated albumin at 4 weeks correlates with A1C levels at 12 weeks and reflects short-term glucose fluctuations. Endocr Pract. 2015;21:1195-1203. 